\title{
Effect of temporary school closure due to COVID-19 on musculoskeletal function in elementary school children
}

\author{
Ryoichi Nakajima1, 2, Hiroshi Kamada ${ }^{2,3}$, Taishu Kasai ${ }^{4}$, Yohei Tomaru ${ }^{3}$, Miki Waku, \\ Ayano Yamaki ${ }^{4}$, Atsuhiko Ban ${ }^{6}$, Shumpei Miyakawa ${ }^{3}$, Masashi Yamazaki ${ }^{2,3}$ and \\ Hitoshi Shiraki \\ ${ }^{1}$ Graduate School of Comprehensive Human Sciences, University of Tsukuba, Japan \\ ${ }^{2}$ Center for Sports Medicine and Health Science, University of Tsukuba Hospital, Japan \\ ${ }^{3}$ Department of Orthopaedic Surgery, Faculty of Medicine, University of Tsukuba, Japan \\ ${ }^{4}$ Faculty of Medicine, University of Tsukuba, Japan \\ ${ }^{5}$ University of Tsukuba Sports Association, Japan \\ ${ }^{6}$ Graduate School of Comprehensive Human Sciences Master's Program in Medical Sciences, University of Tsukuba, Japan \\ ${ }^{7}$ Faculty of Health and Sports Sciences, University of Tsukuba, Japan
}

\begin{abstract}
Objective: In 2020, coronavirus disease-2019 (COVID-19) became the cause of a pandemic. In response, the Japan Sports Agency issued warnings about secondary damage to health, such as the threat to physical and mental well-being due to the lack of exercise in this situation. In this study, we report on cross-sectional and longitudinal examinations of standing trunk flexion to evaluate how temporary long-term school closures affected musculoskeletal function in elementary school students.

Patients and Methods: All children in one public elementary school in T-city during the school years 2019 and 2020 were included in this study. A digital forward flexion meter was used to measure standing trunk flexion.

Results: In this study, 284 (284/289: 98.3\%) and 266 (266/274: 97.1\%) children in school years 2020 and 2019, respectively, were found to have valid data for cross-sectional analysis. The standing trunk flexion did not show significant differences between grades or sexes. In the longitudinal analysis, the results of the comparison of standing trunk flexion in children for two consecutive years revealed significant differences only between grades 3 and $4(P<0.05)$ and between girls in grades 3 and $4(P<0.01)$, but no significant differences in other grades or among boys or girls were observed.

Conclusion: Initially, we expected that there would be a difference in the results of functional assessment using standing trunk flexion depending on the period of absence from school. However, the results of this study showed no significant changes in standing trunk flexion. Moreover, since children's musculoskeletal functions may be affected by various factors during the COVID-19 pandemic, they should be carefully monitored in the future.
\end{abstract}

Key words: standing trunk flexion, COVID-19, children, school closure

(J Rural Med 2021; 16(3): 154-159)

\section{Introduction}

In 2020, coronavirus disease-2019 (COVID-19) caused

Received: February 19, 2021

Accepted: March 30, 2021

Correspondence: Hiroshi Kamada, Center for Sports Medicine and Health Science, University of Tsukuba Hospital, 2-1-1 Amakubo, Tsukuba, Ibaraki 305-8576, Japan

E-mail: hkamada@md.tsukuba.ac.jp

This is an open-access article distributed under the terms of the Creative Commons Attribution Non-Commercial No Derivatives (by-nc-nd) License $<\mathrm{http}: / /$ creativecommons.org/ licenses/by-nc-nd/4.0/>. a worldwide pandemic. In Japan, the Prime Minister issued an emergency declaration in April, and various strategies were implemented, such as requesting the population to refrain from going out ${ }^{1)}$. The entire population had to change to a "new lifestyle" to prevent the spread of COVID-19, and social life has also changed drastically.

In response, the Japan Sports Agency issued warnings about secondary damage to health, such as the threat to physical and mental health due to the lack of exercise in the current situation. The influence on the musculoskeletal system is considered immeasurable ${ }^{2}$ because of the decrease in physical activity due to the promotion of staying at home and teleworking, onset of lifestyle-related diseases in 
middle-aged and older people, decline in physical strength and life functions (deterioration of musculoskeletal organs, such as bones and muscle, and dementia), and risk to healthy growth and development in children.

In view of the situation children are facing, the Ministry of Education, Culture, Sports, Science and Technology issued a notice ${ }^{3)}$ on February 27 directing the "simultaneous temporary closure of elementary schools, junior high schools, senior high schools, and special needs schools to counter the spread of COVID-19", leading to the temporary closure of many schools. In I Prefecture's T-city, the temporary closure began on March 6 , and schools were closed until June 8, so students were unable to go to school for approximately 3 months.

In this context, we aimed to evaluate how temporary long-term school closures affected the musculoskeletal functions of elementary school students. In this study, we selected an objective index, the "standing trunk flexion test", which is one of the recommended items for musculoskeletal examination in school ${ }^{4)}$. We also report on the results of the cross-sectional and longitudinal examinations of this measurement.

\section{Patients and Methods}

\section{Subjects}

All children attending a public elementary school in Tcity, I prefecture, in the school years 2020 and 2019 were included in this study. This school routinely conducts an annual musculoskeletal examination in collaboration with T-city ${ }^{4-6)}$. During the implementation of the musculoskeletal examination, the results of the standing trunk flexion test were used, as it has been measured annually in recent years along with other musculoskeletal parameters. However, the measurement period was different in 2020 because of the temporary closure of schools due to COVID-19, and the measurement was conducted in June, although the measurement is usually conducted in April every year. Students who were absent and those who could not perform the measurement procedure due to trauma or disability were excluded.

\section{Methods}

A digital forward flexion meter (Flection D-, Takei Instruments Co., Ltd.) was used to measure standing trunk flexion. Participants were instructed to stand on the measurement table with their feet together, in line with the reference line, bring their palms together while extending their fingertips, bend their upper body forward, and press the cursor along the post. The measurements were carried out while instructing the student to maintain a position that would not cause recoil or bending of the knee; if these movements were observed, the measurement was invalidated, and a new measurement was performed. Measure- ments were performed twice, and the better of the two was recorded as the measured value.

For the cross-sectional analysis, the mean values of standing trunk flexion were measured for every student in the entire school. Data were recorded according to sex, and the proportion of students who had anteflexion less than 0 $\mathrm{cm}$ was also calculated. For the longitudinal study, students in grades 2 to 6 in 2020 were matched and compared with their individual measurements taken in grades 1-5 in 2019.

\section{Statistical analysis}

In the comparison of means for the cross-sectional analysis, the unpaired t-test was used when normality was observed, and the Mann-Whitney U test was used when normality was not observed. The corresponding t-test was used for longitudinal analysis, and when data were not normally distributed, the Wilcoxon signed rank sum test was used. The chi-square test (including Fisher's direct method and Yate's continuous correction) was used to evaluate the proportion of children who had a measurement of less than 0 $\mathrm{cm}$. The significance level of all tests was set at $5 \%$.

\section{Ethical approval}

This study was approved by the ethics committee of our institution (approval no. 678-3) and was performed in accordance with the Declaration of Helsinki. Written informed consent was obtained from the subjects and their guardians.

\section{Results}

In this study, 284 (284/289: 98.3\%) and 266 (266/274: $97.1 \%$ ) children in the school years 2020 and 2019, respectively, were found to have valid data for cross-sectional study. The overall and sex-specific numbers and proportions for each grade for each year are shown in Table 1. For the longitudinal analysis, 215 of 227 children in grades 2-6 in 2020 were found to have valid data that could be compared with their 2019 data. The number and proportion of students in each year for the entire grade and those by sex are shown in Table 2.

The results of the cross-sectional analysis of standing trunk flexion measurements for each year for all grades and for each sex are shown in Figure 1. A comparison of standing trunk flexion measurements in school years 2020 and 2019 showed no significant differences between grades or sexes. Figure 2 shows the proportion of students with anteflexion of less than $0 \mathrm{~cm}$. No significant difference was noted in the proportion of students with anteflexion of less than $0 \mathrm{~cm}$ between the 2019 and 2020 data for all grades and for each sex.

The results of the longitudinal analysis of the standing trunk flexion measurements for each year for all grades and sex are shown in Figure 3. The results of the comparison 


\section{Journal of Rural Medicine}

Table 1 Number of students with available 2019 and 2020 physical examination data

\begin{tabular}{lllllllll}
\hline & & 1st-grade & 2nd-grade & 3rd-grade & 4th-grade & 5th-grade & 6th-grade & All \\
\hline 2020 & All & 58 & 52 & 53 & 40 & 35 & 45 & 283 \\
& Boys & $28(48 \%)$ & $29(56 \%)$ & $26(49 \%)$ & $22(55 \%)$ & $17(49 \%)$ & $19(42 \%)$ & 141 \\
& Girls & $30(52 \%)$ & $23(44 \%)$ & $27(51 \%)$ & $18(45 \%)$ & $18(51 \%)$ & $26(58 \%)$ & 142 \\
\hline \multirow{2}{*}{2019} & All & 52 & 50 & 41 & 33 & 46 & 44 & 266 \\
& Boys & $30(58 \%)$ & $25(50 \%)$ & $22(54 \%)$ & $17(52 \%)$ & $21(46 \%)$ & $30(68 \%)$ & 145 \\
& Girls & $22(42 \%)$ & $25(50 \%)$ & $19(46 \%)$ & $16(48 \%)$ & $25(54 \%)$ & $14(32 \%)$ & 121 \\
\hline
\end{tabular}

Table 2 Number of students with available 2019 and 2020 physical examination data who could be measured consecutively

\begin{tabular}{llcccccr}
\hline & & 1st-2nd grade & 2nd-3rd grade & 3rd-4th grade & 4th-5th grade & 5th-6th grade & All \\
\hline 2020 & All & 50 & 47 & 40 & 33 & 45 & 215 \\
& Boys & $29(58 \%)$ & $24(51 \%)$ & $22(55 \%)$ & $17(52 \%)$ & $20(44 \%)$ & 112 \\
& Girls & $21(42 \%)$ & $23(49 \%)$ & $18(45 \%)$ & $16(48 \%)$ & $25(56 \%)$ & 103 \\
\hline
\end{tabular}

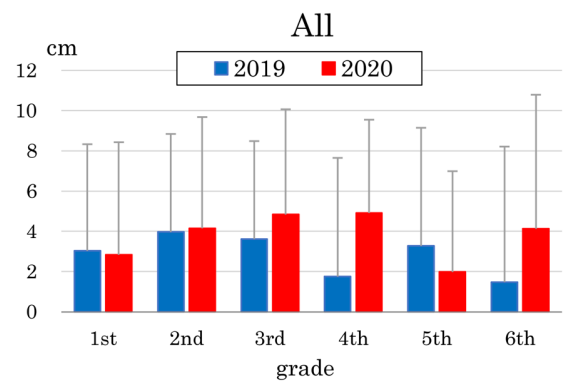

Boys

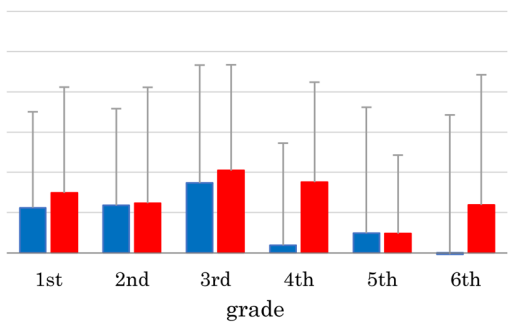

Girls

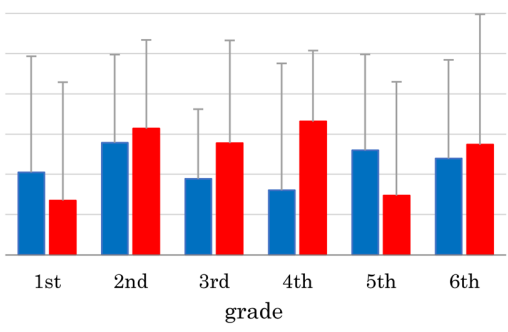

Figure 1 Results of the cross-sectional analysis of standing trunk flexion measurements for school years 2019 and 2020
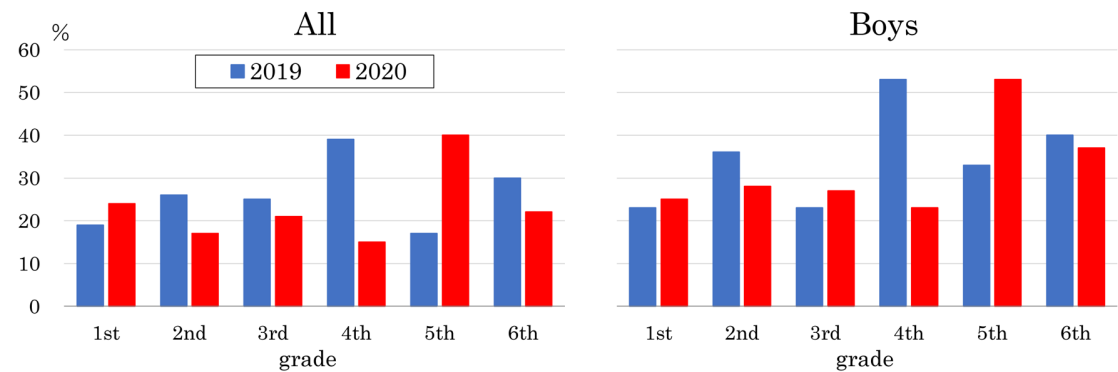

Girls

Figure 2 Results of the cross-sectional analysis of students with an anteflexion of less than $0 \mathrm{~cm}$

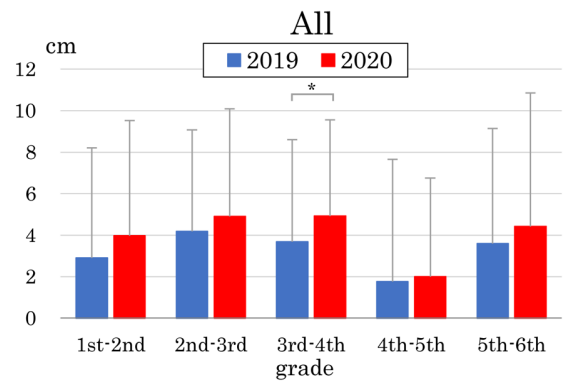

Boys

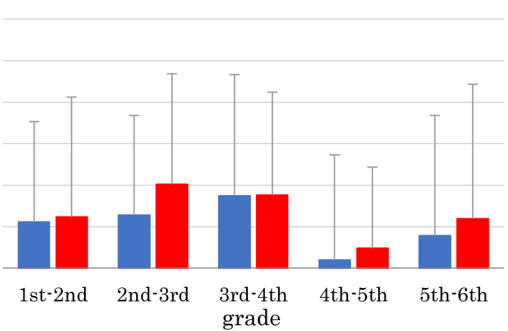

Girls

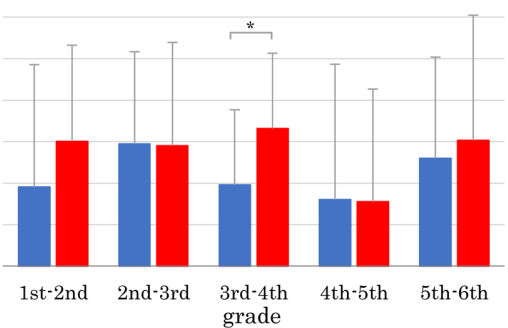

Figure 3 Results of the longitudinal analysis of standing trunk flexion measurements for school years 2019 and $2020\left({ }^{*} P<0.05\right)$ 

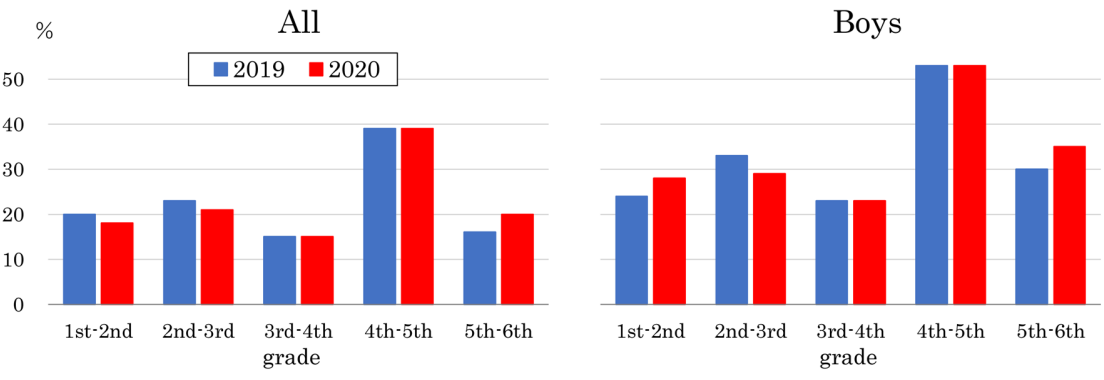

Girls

Figure 4 Results of the longitudinal analysis of the proportion of students with an anteflexion of less than $0 \mathrm{~cm}$

between the 2019 and 2020 standing trunk flexion measurements showed significant differences only in grades 3-4 $(P<0.05)$ and for girls in grades 3-4 $(P<0.01)$, with no significant differences for all grades in boys or girls. The proportion of students with anteflexion of less than $0 \mathrm{~cm}$ is shown in Figure 4. No significant difference was found in the proportion of students with a standing trunk flexion measurement of less than $0 \mathrm{~cm}$ between 2019 and 2020 data for all grades or each sex.

\section{Discussion}

In 2020, public social life has changed drastically due to the spread of COVID-19. While there are concerns about direct effects on health caused by the infection, it is necessary to consider the secondary effects resulting from environmental changes. In this study, we focused on the changes in musculoskeletal function in children due to the spread of COVID-19 infection, which has been reported by the Japan Sports Agency2), and examined the changes based on objective indicators.

In Japan, school physical examinations have been conducted in elementary and junior high schools for many years, and we are grateful for having a system in which children's health conditions are regularly checked annually ${ }^{4,7)}$. Furthermore, in accordance with the partial revision of the enforcement regulations of the School Health and Safety Act, "musculoskeletal examination", which requires the evaluation of the condition of the extremities, has been implemented nationwide simultaneously since 2016. Efforts have been made to detect disorders and diseases at an early stage, leading to early treatment and prevention of musculoskeletal dysfunction. Five years have passed since the start of musculoskeletal examinations, and several reports on musculoskeletal functions have been published. Based on the report that many findings are related to flexibility, such as standing trunk flexion and limited range of motion of the ankle joint ${ }^{8)}$, and on the results of our study ${ }^{6,9,10)}$, we thought that the standing trunk flexion test, which is one of the recommended items of the checklist for musculoskeletal examination, could be an objective indicator of musculo- skeletal function. Initially, we expected that there would be a difference in the results of the functional assessment of standing trunk flexion depending on the period of absence from school or relative inactivity. However, the results of this study showed no significant changes in the assessment of standing trunk flexion in the cross-sectional analysis, and only some girls showed a change in the longitudinal analysis.

Many studies have reported that physical activity and physical fitness are positively correlated in elementary school children and that the sit-and-reach test and activity energy consumption are also correlated ${ }^{11)}$, and Japanese elementary school children are less physically active during the summer vacation than during normal school life ${ }^{12}$. In our preliminary study, we also found that stretching exercises improve flexibility, but flexibility tended to be lower when measured immediately after the summer vacation. At the time of the Great East Japan Earthquake of 2011, which was an unprecedented disaster, a comparison of physical fitness before and after the earthquake showed that physical fitness and exercise ability declined after the earthquake ${ }^{13)}$. From these results, we thought that there might be a reduction in the flexibility of elementary school students when the amount of physical activity decreased because of the temporary closure of schools.

However, in the present study, no decline in standing trunk flexion was observed, and the results showed that the standing trunk flexion of girls in some grades improved. In the physical fitness test currently conducted in elementary schools, the sit-and-reach test is used as an index of flexibility, and this difference in measurement method may have affected the results.

In addition, flexibility is only a part of the physical fitness test and is insufficient to evaluate musculoskeletal function; thus, other indices should be included in the study. Another study after the Great East Japan Earthquake reported that the results of the sit-and-reach test improved after the earthquake, which was due to the use of more mat exercises and flexibility exercises in a limited indoor space due to restrictions on outdoor activities ${ }^{14)}$.

During this temporary holiday period, it is thought that 
the so-called home time increased and more time could be spent freely. The Sports Agency is concerned about the secondary damage to health caused by a lack of exercise ${ }^{2)}$ in this "new lifestyle" and has introduced suggestions for engaging in safe and secure exercise and sports, as well as examples of easy exercise. Children had limited time to be active in their normal school and social lives, but during the temporary closure of schools, they may have had more time to exercise, and the environment for exercise may have been secured by the introduction of methods and increased parental intervention time due to the increase in teleworking. This stay-at-home pattern may lead to an increase rather than a decrease in flexibility, but this is merely speculation.

This study has several limitations. First, the study was limited to one elementary school. Environmental status may vary depending on the individual schools, regions, and backgrounds. It is necessary to increase the number of target schools and students and analyze various data. Second, the different timing of the measurements may have influenced the results, as the temporary school closure due to COVID-19 resulted in a difference of approximately 2 months between the implementation period before 2019 and 2020 . The comparison was possibly not completely accurate because of changes in children's activities and growth. Third, we were unable to assess the amount of physical activity of each student. Individual differences exist in the amount of activity even during periods of absence from school, and it cannot be said that the amount of activity has necessarily decreased. In the future, it is necessary to examine the relationship between the amount of physical activity and exercise habits. Fourth, although it has been argued that standing forward bending is affected by lower limb length ${ }^{15}$, this study did not sufficiently analyze the relationship of individual developmental status, including body size factors such as developmental spurts, and this needs to be examined in the future ${ }^{16-18)}$. In this study, only standing trunk flexion was used as an indicator, but since the school also conducts physical fitness tests on an ongoing basis, we would like to use these results to examine the influence of the absence period on children's musculoskeletal function.

\section{Conclusion}

The effect of COVID-19 on the musculoskeletal function of elementary school students was examined by measuring standing trunk flexion. No changes were observed in crosssectional or longitudinal analyses. However, since children's musculoskeletal functions may change due to various factors during the COVID-19 pandemic, they should be carefully monitored in the future.

Funding: This work was supported by JSPS KAKENHI (grant number 19K11572).

Conflict of interest: The authors declare no conflict of interest associated with this manuscript.

\section{Acknowledgments}

We would like to thank the children and parents of TCity. We would also like to thank the school nurses for their cooperation. We would like to thank Editage (www.editage. com) for English language editing.

\section{References}

1. Cabinet Office Government of Japan, Emergency economic measures to cope with COVID-19. https://www5.cao.go.jp/keizai1/keizaitaisaku/2020/20200420_economic_measures_all.pdf. (April 7, 2020, Amended on April 20, 2020).

2. Japan Sports Agency Countermeasures against infection by the COVID-19. Cautions for sports and exercise. https://www.mext.go.jp/sports/b_menu/ sports/mcatetop05/jsa_00010.html\#002. (Accessed December, 2020).

3. Ministry of Education Culture, Sports, Science and Technology, JAPAN, Simultaneous temporary closure of elementary schools, junior high schools, senior high schools, and special support schools for countermeasures against COVID-19 (Notice). https://www.mext.go.jp/content/202002228-mxt_ kouhou01-000004520 1.pdf, (Accessed March, 2020).

4. Kamada H, Tomaru Y, Kimura M, et al. Four-year musculoskeletal examinations among elementary and junior high school students across a single city. J Rural Med 2020; 15: 194-200. [Medline] [CrossRef]

5. Tomaru Y, Kamada H, Tsukagoshi Y, et al. Screening for musculoskeletal problems in children using a questionnaire. J Orthop Sci 2019; 24: 159-165. [Medline] [CrossRef]

6. Kasai T, Kamada H, Tomaru Y, et al. Longitudinal changes in musculoskeletal findings of elementary and junior high school students: a 1-year prospective study. J Phys Fit Sports Med 2020; 9: 53-64. [CrossRef]

7. Yamaguchi N, Chosa E, Yamamoto K, et al. Screening for musculoskeletal problems in Japanese schoolchildren: a cross-sectional study nested in a cohort. Public Health 2016; 139: 189-197. [Medline] [CrossRef]

8. Morihara T, Tachiiri K, Fukuda K, et al. Locomotive screening in Kyoto - Screening of functional disorder of locomotion using arm elevation, trunk flexion and squatting-. J Musculoskelet Med 2014; 25: 225-230 (in Japanese, Abstract in English).

9. Tomaru Y, Kamada H, Tsukagoshi Y, et al. Relationship between exercise time and musculoskeletal problems in children. J Rural Med 2019; 14: 176-180. [Medline] [CrossRef]

10. Tomaru Y, Kamada H, Tsukagoshi Y, et al. Determining the relationship between physical status and musculoskeletal injuries in children: a cohort study. J 
Rural Med 2020; 15: 116-123. [Medline] [CrossRef]

11. Niimoto S, Yamasaki M. The relationship between physical strength and physical activity in elementary school children. Japan Journal of Human Growth and Development Research 2013; 61: 9-18 (in Japanese, Abstract in English). [CrossRef]

12. Tanaka C, Reilly JJ, Tanaka M, et al. Seasonal changes in objectively measured sedentary behavior and physical activity in Japanese primary school children. BMC Public Health 2016; 16: 969. [Medline] [CrossRef]

13. Kikuchi S. The impact of environmental changes after the Great East Japan Earthquake on children. Hoken no. Kagaku 2019; 61: 559-565 (in Japanese).

14. Ogawa H, Kanke R. The change of physical and motor ability of school children in Fukushima before and after the earthquake disaster of 2011: A comparison of the results of physical and motor ability tests performed in fiscal year 2010 and 2011. Japanese Journal of Sport Education Studies 2018; 37: 1-14 (in Japanese, Abstract in English). [CrossRef]

15. Oyama Y. A hygienic study on the flexibility of the normal. 1. The measurement methodology. Nippon Eiseigaku Zasshi 1965; 20: 65-70 (in Japanese, Abstract in English). [Medline] [CrossRef]

16. Csukás A, Takai S, Baran S. Adolescent growth in main somatometric traits of Japanese boys: Ogi Longitudinal Growth Study. Homo 2006; 57: 73-86. [Medline] [CrossRef]

17. Hattori K, Hirohara T, Satake T. Body proportion chart for evaluating changes in stature, sitting height and leg length in children and adolescents. Ann Hum Biol 2011; 38: 556-560. [Medline] [CrossRef]

18. Nakata H, Akido M, Naruse K, et al. Relative age effect in physical fitness among elementary and junior high school students. Percept Mot Skills 2017; 124: 900-911. [Medline] [CrossRef] 